\title{
Exacerbation of symptoms due to diltiazem in a patient with Lambert- Eaton myasthenic myopathic syndrome
}

\section{KD Pathirana ${ }^{1}$ and MDP Hidelaratchi ${ }^{2}$}

(Index words: Calcium channel blockers, diltiazem, Lambert-Eaton myasthenic syndrome)

\begin{abstract}
A 65-year old man on treatment for ischaemic heart disease presented with a history of pain and weakness of all four limbs and dry mouth. He had clinical and neurophysiological features of Lambert-Eaton myasthenic syndrome. His symptoms improved after withdrawal of diltiazem, although neurophysiological changes remained even after 3 months.
\end{abstract}

\section{Case report}

A 65-year old retired teacher presented with progressive difficulty in raising his arms, difficulty in rising from the squatting position and pain in arms and legs of 3 months. He complained of dry mouth for four years. He denied any postural giddiness, bladder symptoms or impotence. He was on treatment for hypercholesterolaemia and ischaemic heart disease (IHD) with diltiazem $90 \mathrm{mg}$ in three divided doses, atenolol $50 \mathrm{mg}$, aspirin $150 \mathrm{mg}$, and lovastatin $10 \mathrm{mg}$ once daily. He was awaiting coronary artery bypass surgery. He was a teetotaller and had not smoked for 15 years.

On examination he had mild wasting and grade 4 weakness of proximal girdle muscles with fatigability. Tendon reflexes were absent but augmented with repeated tapping and after isometric contraction of the muscles. The rest of the nervous system examination and examination of the other systems were unremarkable.

The following investigations were normal: serum electrolytes, urinanalysis, fasting blood glucose, blood urea and electrolytes, thyroid function tests, ESR, serum creatine kinase (CPK), aldolase, serum calcium, liver function tests. Chest radiograph, CT scan of chest and ultrasound scan of the abdomen did not reveal evidence of an underlying malignancy. Electron microscopy of the muscle biopsy too was normal. Edrophonium test was positive with improvement of proximal muscle power with the drug but not with placebo.

\section{Neurophysiology}

The needle EMG was normal. Stimulation of the right median nerve yielded a compound muscle action potential (CMAP) with small amplitude, which increased by $>200 \%$ after isometric stimulation (post-exercise facilitation) and reduced to $21 \%$ after rest (Figure 1). Repetitive stimulation of the median nerve at $3 \mathrm{~Hz}$ did not show a significant change; at $20 \mathrm{~Hz}$ a decrement was shown while at $50 \mathrm{~Hz}$ a more than $300 \%$ increment response was seen (Figure 2). These finding were compatible with Lambert-Eaton myastheric syndrome (LEMS).

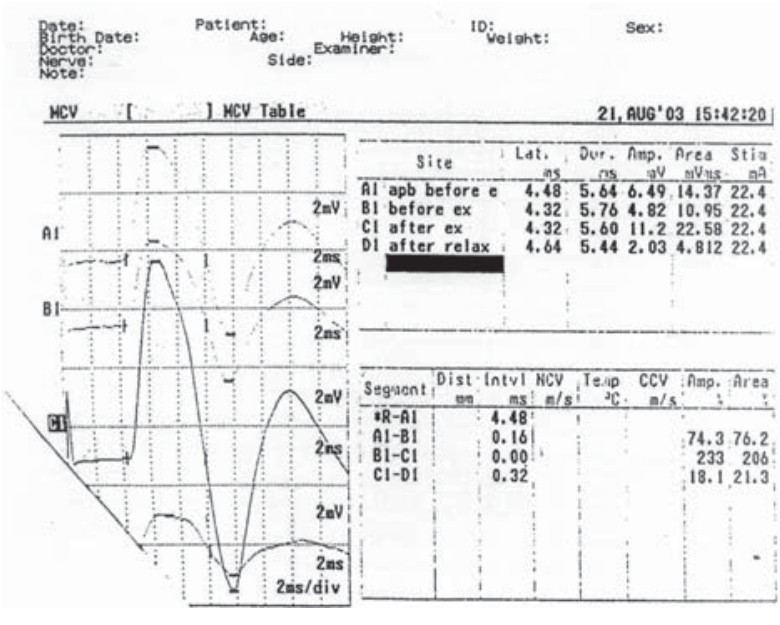

Figure 1. Compound muscle action potentials of the right median nerve before and after isometric stimulation.

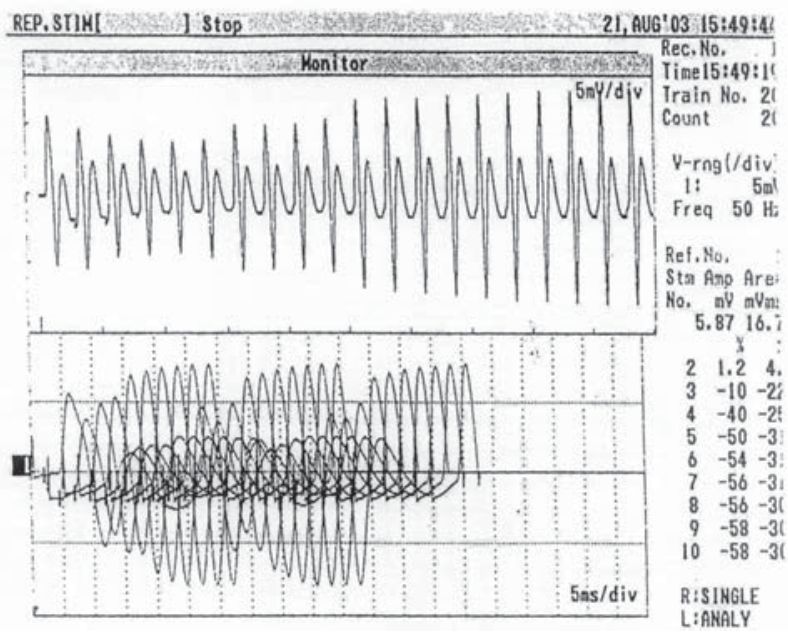

Figure 2. Repetitive stimulation of median nerve at $3 \mathrm{~Hz}$, $20 \mathrm{~Hz}$ and $50 \mathrm{~Hz}$.

On admission, lovastatin was stopped considering the possibility of statin induced myositis. Due to lack of 
improvement in symptoms after its withdrawal and normal CPK, lovastatin was reintroduced.

Diltiazem was stopped after confirmation of LEMS and the patient was reassessed after 3 months. At this stage he had partial improvement of the symptoms, though the neurophysiological studies revealed the same changes. He was then treated with pyridostigmine as 2, 4 diaminopyridine was not available. He was free of symptoms without any drugs 6 months after withdrawing diltiazem. As he did not consent for further neurophysiological tests, a continuous follow up and periodic reassessment to screen for any underlying malignancy is planned.

\section{Discussion}

Lambert-Eaton myasthenic syndrome is an uncommon disorder of the neuromuscular junction, caused by antibodies against voltage-gated calcium channels (VGCC) of the presynaptic nerve endings. It can be broadly divided into two groups - those associated with malignancy and those without association. The commonly encountered carcinoma is the small cell carcinoma of the lung (SCLC) which accounts to about $60 \%$ of the cases, while lymphoproliferative disorders, carcinoma of the breast, colon, pancreas, prostate are rare associations [1]. In a patient presenting with LEMS the probability of having a SCLC falls significantly after 2 years, while it is almost negligible after 4-5 years [2]. This category is considered to be the idiopathic group.

The classical presentation of LEMS is with proximal myopathy and autonomic symptoms such as dry mouth, impotence and bladder or bowel symptoms. LEMS associated with carcinoma presents at a later age (mean 57.9 years), while those without, present relatively early (mean 48 years). In both groups a male preponderance is seen [3].

The release of neurotransmitters at the presynaptic motor nerve terminals of the neuromuscular junction and autonomic neurons depends on the influx of calcium through the VGCC. Over $90 \%$ of patients in both groups of LEMS have antibodies against P/Q type VGCCs [4, 5], though it has a stronger bearing to carcinoma associated LEMS.

Calcium channel blockers are known to interact with VGCCs in vitro [6]. After an extensive search in literature we found only a few reports of calcium channel blockers precipitating symptoms of neuromuscular junction disorders. Felodipine, nifedipine and verapamil exacerbating symptoms of myasthenia gravis and possible LEMS have been previously reported [7]. Ueno and Hara have reported a 59-year old man with IHD who had developed clinical and electromyographic changes of LEMS following treatment with diltiazem which improved clinically and neurophysiologically after withdrawal of the drug [8].

Our patient fulfills the neurophysiological criteria to confirm the diagnosis of LEMS. Improvement of symptoms but not neurophysiological features probably indicates that this is a case of LEMS exacerbated by diltiazem, rather than LEMS caused by diltiazem, as proposed by Ueno and Hara. Hence there is a need for follow up of this patient for an underlying malignancy at least for 5 years to detect the same.

\section{Acknowledgements}

We thank Dr. D Idampitiya and Dr. C Idampitiya for the literature search.

\section{References}

1. Seneviratne U, de Silva R. Lambert-Eaton myasthenic syndrome. Postgraduate Medical Journal 1999; 75: 516-20.

2. O'Neill JH, Murray NM, Newsom-Davis J. The LambertEaton myasthenic syndrome. A review of 50 cases. Brain 1988; 111: 577-96.

3. Gutmann L, Philips LH, Gutmann L. Trends in the association of Lambert-Eaton myasthenic syndrome with carcinoma. Neurology 1992; 42: 848-50.

4. Lennon VA, Kryzer TJ, Griesmann GE, O'Suilleabhain PE, Windebank AJ, et al. Calcium-channel antibodies in the Lambert-Eaton syndrome and other paraneoplastic syndromes. New England Journal of Medicine 1995; 332: 1467-75.

5. Motomura M, Lang B, Johnston I, Palace J, Vincent A, et al. Incidence of serum anti-P/Q -type and anti-N-type calcium channel autoantibodies in the Lambert-Eaton myasthenic syndrome. Journal of Neurological Sciences 1997; 147: 35-42.

6. Neuromuscular syndromes. www.neuro.wustl.edu/ neuromuscular.

7. Pina Latorre M A, Cobeta J C, Rodilla F, Navarro N, Zabala S. Influence of calcium antagonist drugs in myasthenia gravis in the elderly. Journal of Clinical Pharmacy and Therapeutics 1998; 23: 399-403.

8. Ueno S, Hara Y. Lambert-Eaton myasthenic syndrome without anti-calcium channel antibody: adverse effect of calcium antagonist diltiazem. Journal of Neurology, Neurosurgery and Psychiatry. 1992; 55: 409-10. 